\title{
Propagation of Uncertainty in Cooperative Multirobot Localization: Analysis and Experimental Results
}

\author{
Stergios I. Roumeliotis* \\ Department of Computer Science $\&$ Engineering, University of Minnesota \\ Ioannis M. Rekleitis ${ }^{\dagger}$ \\ Mechanical Engineering, Carnegie Mellon University
}

\begin{abstract}
This paper examines the problem of cooperative localization for the case of large groups of mobile robots. A Kalman filter estimator is implemented and tested for this purpose. The focus of this paper is to examine the effect on localization accuracy of the number $N$ of participating robots and the accuracy of the sensors employed. More specifically, we investigate the improvement in localization accuracy per additional robot as the size of the team increases. Furthermore, we provide an analytical expression for the upper bound on the positioning uncertainty increase rate for a team of $N$ robots as a function of $N$, the odometric and orientation uncertainty for the robots, and the accuracy of a robot tracker measuring relative positions between pairs of robots. The analytical results derived in this paper are validated both in simulation and experimentally for different test cases.
\end{abstract}

Keywords: Multirobot Localization, Cooperative Localization, Distributed Sensing, Networks of Robots.

\section{Introduction}

This paper studies the localization accuracy of a team of mobile robots that closely cooperate while navigating within the same area. The problem of localization is central in mobile robotics. One of the advantages of multi-robot systems is that robots can accurately localize by measuring their relative position and/or orientation and communicating localization information throughout the group. Although external positioning information from a GPS receiver or a map of the environment can further increase the overall localization accuracy, we hereafter consider primarily the most challenging scenario where the absolute positions of the robots cannot be measured or inferred. In this case the uncertainty in the position estimates for all robots will continuously increase. Previous work on cooperative localization [18, 23, 29] has demonstrated that the localization uncertainty increase across groups of robots is lower compared to the situation where each robot is estimating its position without cooperation with the rest of the team.

\footnotetext{
* Contact author for correspondence. Email: stergios@cs.umn.edu

† Email: rekleitis@cmu.edu
} 
The theoretical analysis of the positioning uncertainty propagation during cooperative localization has been an open problem to this date. In this paper we present the first theoretical treatment for determining upper bounds on the position uncertainty accumulation for a group of $N$ robots by directly solving the continuous time Riccati equation for the covariance of the errors in the position estimates. The key element in our derivation is the separation of the covariance matrix into two sets of submatrices: the ones that converge to steady state values and those that capture the time dependence of the uncertainty increase during cooperative localization.

The main focus is on homogeneous teams of robots, i.e., groups of robots that have the same proprioceptive (odometric in this case) and exteroceptive (orientation and relative position) sensing capabilities. Nevertheless, the derived expressions are also applicable for determining the upper localization uncertainty bounds for heterogeneous teams of robots based on the sensing capabilities of the robot with the least accurate sensors within the team. The resulting formulae are for the maximum expected uncertainty. Since both the kinematics of the robots and the relative position measurements in $2 \mathrm{D}$ are described by sets of nonlinear and time-varying equations, in this treatment we consider maximum expected values for the covariances of the different sources of uncertainty and noise [28].

Throughout the paper we assume that all robots move at the same time randomly. Each robot continuously measures the relative position of every other robot in the team. Moreover, each robot is equipped with a sensor (such as a compass, or, sun sensor) of limited accuracy that provides absolute orientation measurements. This is required in the derivations that follow for determining bounds on the orientation uncertainty for each robot. If such sensor is not available, then an upper bound for the orientation uncertainty needs to be defined by alternative means, e.g., by estimating orientation from the structure of the environment around the robot $[19,22]$, or by deriving an estimate for the maximum orientation uncertainty from odometry over a certain period of time for each robot [14]. In these cases the resulting expressions will provide an upper bound for the localization uncertainty in the group.

In the following section we outline the main approaches to cooperative localization. In Section 3, we present the formulation of the multi-robot localization problem and study the effect of consecutive relative position updates on the structure of the Riccati equation describing the time evolution of the uncertainty in the position estimates. Section 4 contains the derivations of the analytical expression for the uncertainty propagation in the case of cooperative localization. In Sections 5 and 6 , experimental and simulation results are presented that 
validate the derived analytical expressions for the rate of localization increase. Finally, Section 7 draws the conclusions from this analysis and suggests directions of future work.

\section{Related Work}

Many robotic applications require that robots work in collaboration [20] in order to perform a certain task $[1,5,6,21,33]$. When a group of robots needs to coordinate efficiently, precise localization is of critical importance. In these cases, multi-robot cooperation for determining positioning estimates will result in better localization by compensating for errors in odometry and/or a pose sensor.

Previous work on multiple robots has considered collaborative strategies when lack of landmarks made localization impossible [4]. An example of a system designed for cooperative localization was first reported in [18]. A group of robots is divided into two teams in order to perform cooperative positioning. At each instant, one team is in motion while the other team remains stationary and acts as a landmark. The teams then exchange roles and the process continues until both teams have reached their target. Improvements over this system and optimum motion strategies are discussed in [17, 15, 16]. Similarly, in [11], only one robot moves, while the rest of the team of small-sized robots forms an equilateral triangle of localization beacons in order to update their pose estimates. Another implementation of cooperative localization is described in $[23,24]$. In this approach a team of robots moves through the free space systematically mapping the environment. At each time instant at least one robot is stationary acting as a landmark for the localization of the moving robots. Furthermore, the moving robots, by maintaining an uninterrupted line of visual contact, ensure that the area between the stationary and the moving robots is free of obstacles. All previous approaches have the following limitations: (a) Only one robot (or team) is allowed to move at any given time, and (b) The two robots (or teams) must maintain visual (or sonar) contact at all times.

A different collaborative multirobot localization schema is presented in $[7,8]$. The authors have extended the Monte Carlo localization algorithm [32] to the case of two robots when a map of the area is available to both robots. When these robots detect each other, the combination of their belief functions facilitates their global localization task. The main limitation of this approach is that it can be applied only within known indoor environments. In addition, since information interdependencies are being ignored every time the two robots meet, this method can lead to overly optimistic position estimates. This issue is discussed in detail 
in [27]. A particle filter was also used in the context of multi-robot exploration in [26].

A Kalman filter based implementation of a cooperative navigation schema is described in [31]. In this case the effect of the orientation uncertainty in both the state propagation and the relative position measurements is ignored resulting in a simplified distributed algorithm. In $[29,30]$ a Kalman filter pose estimator is presented for a group of simultaneously moving robots. Each of the robots collects sensor data regarding its own motion and shares this information with the rest of the team during the update cycles. The Kalman filter is decomposed into a number of smaller communicating filters, one for every robot, processing sensor data collected by its host robot. It has been shown that when every robot senses and communicates with its colleagues at all times, every member of the group has less uncertainty about its position than the robot with the best (single) localization results. Finally, in [12] an alternative to the Kalman filter approach was presented. A Maximum Likelihood estimator was formulated to process relative pose and odometric measurements recorded by the robots and a solution was derived by invoking numerical optimization.

Different sensing modalities have been used by teams of mobile robots in order to track each other. Some sensors are able to estimate accurately the distance between robots, such as the ultrasound wave used in the milibots project [10]. Other sensors estimate the bearing of the observed robot such as the omnidirectional video cameras used in $[13,9]$, or both the distance and relative bearing, with stereo vision and active lighting in [3], and vision and laser range scanners in [2]. Finally, sensors are able to estimate even the orientation of the observed robot, in addition to the distance and relative bearing [15, 24].

To the best of our knowledge there exist only two cases in the literature where analysis of the uncertainty propagation has been considered in the context of cooperative localization. In [31] the improvement in localization accuracy is computed after only a single update step with respect to the previous values of position uncertainty. In this case the robot orientations are assumed to be perfectly known and no expressions are derived for the propagation of the localization uncertainty with respect to time or the accuracy of the odometric and relative position measurements. In [25] the authors studied in simulation the effect of different robot tracker sensing modalities in the accuracy of cooperative localization. Statistical properties were derived from simulated results for groups of robots of increasing size $N$ when only one robot moved at a time.

Hereafter we present the details of our approach for estimating the uncertainty propagation during cooperative localization. Our initial 
formulation is based on the algorithm described in [30]. The main difference is that the robots instead of measuring their relative orientations, have access to absolute orientation measurements.

\section{Cooperative Localization}

Consider the case of a mobile robot moving on flat terrain and equipped with odometric sensors that measure its linear and rotational velocity. In this case the pose of the robot (in discrete time) is given by

$$
\begin{aligned}
& x(k+1)=x(k)+V(k) \delta t \cos (\phi(k)) \\
& y(k+1)=y(k)+V(k) \delta t \sin (\phi(k)) \\
& \phi(k+1)=\phi(k)+\omega(k) \delta t
\end{aligned}
$$

where $V(k)$ and $\omega(k)$ are the linear and rotational velocity of the robot at time $k$. This non-linear set of equations can be used to propagate the estimate for the pose of the robot as

$$
\begin{aligned}
\hat{x}(k+1 \mid k) & =\hat{x}(k \mid k)+V_{m}(k) \delta t \cos (\hat{\phi}(k \mid k)) \\
\hat{y}(k+1 \mid k) & =\hat{y}(k \mid k)+V_{m}(k) \delta t \sin (\hat{\phi}(k \mid k)) \\
\hat{\phi}(k+1 \mid k) & =\hat{\phi}(k \mid k)+\omega_{m}(k) \delta t
\end{aligned}
$$

where

$$
\begin{aligned}
& V_{m}(k)=V(k)-w_{V}(k) \\
& \omega_{m}(k)=\omega(k)-w_{\omega}(k)
\end{aligned}
$$

are the measured linear and rotational velocity of the robot, and $w_{V}(k)$ $\left(w_{\omega}(k)\right)$ is the noise contaminating the linear (rotational) velocity measurements. Both $w_{V}(k)$ and $w_{\omega}(k)$ are assumed to be independent zero-mean white Gaussian processes with known variances

$$
\sigma_{V}^{2}=E\left\{w_{V}^{2}\right\}, \sigma_{\omega}^{2}=E\left\{w_{\omega}^{2}\right\}
$$

If the robot receives absolute orientation measurements

$$
z(k+1)=\phi(k+1)+n_{\phi}(k+1)
$$

with $n_{\phi}(k+1)$ a zero-mean white Gaussian process with known variance $\sigma_{\phi}^{2}=E\left\{n_{\phi}^{2}\right\}$, these measurements can be processed to improve the odometric estimates $(\hat{x}, \hat{y}, \hat{\phi})$ of the pose of the robot.

Instead of formulating an estimator that combines both odometric and absolute orientation measurements, we follow a two-tier approach to this problem where absolute orientation measurements are 
first combined with odometric measurements of the rotational velocity of each robot independently. Then, the resulting improved orientation estimates are used to propagate the position estimates of the robot. Although this approach is suboptimal compared to an estimation scheme that combines all odometric and absolute orientation measurements at once, it will facilitate the derivation of an analytical expression for bounding the uncertainty in the case of groups of robots moving in 2D. The same two-tier approach can be used to derive similar expressions for motion in 3D.

\subsection{Orientation Estimation}

For the first layer of estimation, the rotational velocity measurements are used to propagate the orientation estimates for the robot. This is described by the following equation: ${ }^{1}$

$$
\begin{aligned}
\dot{\hat{\phi}}(t)=\omega_{m}(t) & =0 \cdot \hat{\phi}(t)+1 \cdot \omega(t)-1 \cdot w_{\omega_{c}}(t) \\
& =F \cdot \hat{\phi}(t)+B \cdot \omega(t)+G_{c} \cdot w_{\omega_{c}}(t)
\end{aligned}
$$

which is the continuous time form of Eq. (3) with $E\left\{w_{\omega_{c}}(t) w_{\omega_{c}}(\tau)\right\}=$ $\sigma_{\omega_{c}}^{2} \delta(t-\tau)=Q_{c}(t) \delta(t-\tau)$. The orientation estimates are then updated by processing the absolute orientation measurements

$$
z(t)=\phi(t)+n_{\phi}(t)
$$

with $E\left\{n_{\phi}(t) n_{\phi}(\tau)\right\}=\sigma_{\phi_{c}}^{2} \delta(t-\tau)=R_{c}(t) \delta(t-\tau)$. In order to estimate the uncertainty of the orientation estimates at steady state we invoke the continuous time Riccati equation

$$
\dot{P}=F P+P F^{T}+G_{c} Q_{c} G_{c}^{T}-P H^{T} R_{c}^{-1} H P
$$

with $P=\sigma_{\phi_{o}}^{2}$ and $H=1$. The previous equation at steady state is:

$$
\lim _{t \rightarrow \infty} \dot{P}=0=\sigma_{\omega_{c}}^{2}-\frac{1}{\sigma_{\phi_{c}}^{2}} \sigma_{\phi_{o c}}^{4} \Rightarrow \sigma_{\phi_{o c}}^{2}=\sigma_{\phi_{c}} \sigma_{\omega_{c}}
$$

This last expression provides the estimate for the uncertainty in the orientation of the robot when rotational velocity and absolute orientation measurements are processed.

\footnotetext{
1 The subscript "c" is used to indicate continuous time quantities.
} 


\subsection{Position Uncertainty Propagation for a Single Robot}

At this point we use this last result to provide an expression for the uncertainty propagation of the position estimates for the case of a single robot. By linearizing the continuous time equivalent of Eqs. (1), (2), the position error propagation equations for the robot can be written in a matrix expression as:

$$
\begin{aligned}
& {\left[\begin{array}{c}
\dot{\tilde{x}}(t) \\
\tilde{\tilde{y}}(t)
\end{array}\right]=\left[\begin{array}{ll}
0 & 0 \\
0 & 0
\end{array}\right]\left[\begin{array}{l}
\tilde{x}(t) \\
\tilde{y}(t)
\end{array}\right]+\left[\begin{array}{cc}
\cos \hat{\phi}(t) & -V_{m}(t) \sin \hat{\phi}(t) \\
\sin \hat{\phi}(t) & V_{m}(t) \cos \hat{\phi}(t)
\end{array}\right]\left[\begin{array}{c}
w_{V_{c}}(t) \\
\tilde{\phi}_{c}(t)
\end{array}\right]} \\
& \Leftrightarrow \dot{\tilde{X}}(t)=F(t) \tilde{X}(t)+G_{c}(t) W_{c}(t)
\end{aligned}
$$

where $F(t)=0_{2 \times 2}$ is a matrix of zeros,

$$
\begin{aligned}
E\left\{G_{c}(t) W_{c}(t) W_{c}^{T}(\tau) G_{c}^{T}(t)\right\} & =G_{c}(t)\left[\begin{array}{cc}
\sigma_{V_{c}}^{2} & 0 \\
0 & \sigma_{\phi_{o c}}^{2}
\end{array}\right] G_{c}^{T}(t) \delta(t-\tau) \\
& =Q_{c}(t) \delta(t-\tau)
\end{aligned}
$$

As evident from the previous expressions, the covariance $Q_{c}(t)$ for all sources of uncertainty and noise during propagation, is a time-varying matrix. The values of the elements of this matrix depend on the measured velocity $V_{m}(t)$ of the robot and the estimate of its orientation $\hat{\phi}(t)$. If we assume that the robot moves with constant velocity $V$ and we average across all possible values of its orientation, the previous covariance matrix is given by:

$$
\bar{Q}_{c}(t)=\frac{\sigma_{V_{c}}^{2}+\sigma_{\phi_{o c}}^{2} V^{2}}{2} I_{2 \times 2}=q_{c} I_{2 \times 2}
$$

When no relative positioning information is available, the covariance for the position of the robot is propagated using only odometric information. This is described by the following equation:

$$
\dot{P}=F P+P F^{T}+\bar{Q}_{c}=\bar{Q}_{c}=q_{c} I
$$

or

$$
P(t)=\left(q_{c} t+p_{0}\right) I
$$

where $p_{0}$ is the initial positioning uncertainty of the robot. As it is evident, the covariance (uncertainty) for the position of a single robot increases, on the average, linearly with time at a rate of $q_{c}$ determined in Eq. (11) that depends on the accuracy of the absolute orientation measurements $\left(\sigma_{\phi_{c}}\right)$ and the robot's odometry $\left(\sigma_{V_{c}}, \sigma_{\omega_{c}}, V\right)$. 


\subsection{Relative Position Measurements}

At this point instead of one robot, we consider the case of a group of robots where each of them (i) estimates its orientation by fusing rotational velocity measurements with absolute orientation measurements, (ii) propagates its position using the previous orientation estimates and linear velocity measurements, and (iii) measures the relative position $z_{i j}$ of all other robots in the team:

$$
z_{i j}(k+1)={ }^{i} \vec{p}_{j}(k+1)+n_{z_{i j}}(k+1)=C^{T}\left(\phi_{i}\right)\left(\vec{p}_{j}-\vec{p}_{i}\right)+n_{z_{i j}}
$$

This additional relative position information can be used to improve the localization accuracy in the group. In the previous equation, $\vec{p}_{i}$ symbolizes the $x_{i}$ and $y_{i}$ coordinates of robot's $i$ position, i.e. $\vec{p}_{i}=\left[\begin{array}{ll}x_{i} & y_{i}\end{array}\right]^{T}$. The preceding superscript denotes the frame of reference $i$ attached on the observing robot $i$ that the position of robot $j$ is expressed to. For notation simplicity, when no preceding superscript appears, this quantity is expressed with respect to the global frame of reference. Finally, $n_{z_{i j}}$ is the noise in the relative position measurement, assumed to be a zero-mean white Gaussian process, and $C(\phi)$ is the rotational matrix:

$$
C(\phi)=\left[\begin{array}{cc}
\cos \phi & -\sin \phi \\
\sin \phi & \cos \phi
\end{array}\right]
$$

By linearizing Eq. (13), the measurement error equation is given by

$$
\begin{aligned}
\widetilde{z}_{i j}(k+1) & =z_{i j}(k+1)-\hat{z}_{i j}(k+1) \\
& =H_{i j}(k+1) \widetilde{X}(k+1)+\Gamma(k+1) N_{i j}(k+1)
\end{aligned}
$$

where

$$
\begin{aligned}
& H_{i j}(k+1)=C^{T}\left(\hat{\phi}_{i}\right)^{o} H_{i j} \\
& { }^{o} H_{i j}=\left[\begin{array}{lllllll}
0 & \ldots & -I & \ldots & I & \ldots & 0
\end{array}\right] \\
& \tilde{X}(k+1)=\left[\begin{array}{lllllll}
\widetilde{p}_{1}^{T} & \ldots & \widetilde{p}_{i}^{T} & \ldots & \widetilde{p}_{j}^{T} & \ldots & \widetilde{p}_{N}^{T}
\end{array}\right]^{T} \\
& \Gamma(k+1)=\left[I-C^{T}\left(\hat{\phi}_{i}(k+1)\right) J \widehat{\Delta p}_{i j}(k+1)\right] \\
& \widehat{\Delta p}_{i j}(k+1)=\hat{p}_{j}(k+1)-\hat{p}_{i}(k+1) \\
& N_{i j}(k+1)=\left[\begin{array}{c}
n_{z_{i j}}(k+1) \\
\widetilde{\phi}_{i}(k+1)
\end{array}\right], \quad J=\left[\begin{array}{cc}
0 & -1 \\
1 & 0
\end{array}\right]
\end{aligned}
$$

The covariance for the measurement error is given by

$$
\begin{aligned}
R_{i j}(k+1) & =\Gamma(k+1) E\left\{N_{i j}(k+1) N_{i j}^{T}(k+1)\right\} \Gamma^{T}(k+1) \\
& =R_{z_{i j}}(k+1)+R_{\tilde{\phi}_{i j}}(k+1)
\end{aligned}
$$


This expression encapsulates all sources of noise and uncertainty that contribute to the measurement error $\widetilde{z}_{i j}$. More specifically, $R_{z_{i j}}$ is the covariance of the noise $n_{z_{i j}}$ in the recorded relative position measurement $z_{i j}$ and $R_{\tilde{\phi}_{i j}}$ is the additional covariance term due to the error $\tilde{\phi}_{i}$ in the orientation estimate $\hat{\phi}_{i}$ of the observing robot $i$.

When each of the $N$ robots measures the relative position of every other robot in the group, the measurement matrix is:

$$
\mathbf{H}^{T}=\left[\begin{array}{lll}
\boldsymbol{H}_{1}^{T} & \ldots & \boldsymbol{H}_{N}^{T}
\end{array}\right]
$$

where $\boldsymbol{H}_{i}$ is the measurement matrix for all the relative position measurements received by robot $i$ :

$$
\boldsymbol{H}_{i}=\left[\begin{array}{c}
H_{i 1} \\
\vdots \\
H_{i N}
\end{array}\right]=\left[\begin{array}{c}
C^{T}\left(\hat{\phi}_{i}\right)^{o} H_{i 1} \\
\vdots \\
C^{T}\left(\hat{\phi}_{i}\right)^{o} H_{i N}
\end{array}\right]=\operatorname{Diag}\left(C^{T}\left(\hat{\phi}_{i}\right)\right)^{o} \boldsymbol{H}_{i}
$$

The total information $\mathbf{H}^{T} \mathbf{R}^{-1} \mathbf{H}$ available to the system is:

$$
\mathbf{H}^{T} \mathbf{R}^{-1} \mathbf{H}=\sum_{i=1}^{N} \boldsymbol{H}_{i}^{T} \boldsymbol{R}_{i}^{-1} \boldsymbol{H}_{i}=\sum_{i=1}^{N}{ }^{o} \boldsymbol{H}_{i}^{T o} \boldsymbol{R}_{i}^{-1 o} \boldsymbol{H}_{i}
$$

Assuming that all robots move within a constrained area, it can be shown [28] that the minimum information available to the system is: ${ }^{2}$

$$
\mathbf{H}^{T} \mathbf{R}^{-1} \mathbf{H}=2 N \mathbf{I}\left({ }^{o} \bar{R}^{-1}\right)-2 \mathbf{1}\left({ }^{o} \bar{R}^{-1}\right)
$$

with

$$
{ }^{o} \bar{R}^{-1}=\frac{1}{{ }^{o} \bar{r}_{z}} I_{2 \times 2} \quad, \quad{ }^{o} \bar{r}_{z}=\max \left(\sigma_{\rho_{c}}^{2}, \rho_{o}^{2} \sigma_{\theta_{c}}^{2}\right)+(N-1) \rho_{o}^{2} \sigma_{\phi_{o c}}^{2}
$$

where $\sigma_{\rho_{c}}^{2}$ and $\sigma_{\theta_{c}}^{2}$ are the variances of the distance and bearing measurements, and $\rho_{o}$ is the maximum distance between any two robots.

In the following section, we solve the Riccati equation for the maximum expected uncertainty for this group of robots when performing cooperative localization [28].

\footnotetext{
${ }^{2}$ From here on, we use the notation $\mathbf{I}(A)$ to denote a matrix composed of $N$ diagonal submatrices $A$ and $N(N-1)$ non-diagonal submatrices 0 and $\mathbf{1}(B)$ to denote a matrix whose all $N^{2}$ submatrix elements are equal to $B$.
} 


\subsection{Consecutive Relative Position Updates}

Assume that at the time step $k=0$ all robots know their position with the same level of accuracy, that is $\mathbf{P}_{0}=\mathbf{I}\left(P_{0}\right), P_{0}=p_{0} I_{2 \times 2}$. The covariance matrix $\mathbf{P}_{0}$ is symmetric with equal non-diagonal submatrices (here all zero) and also equal diagonal submatrices (here all $p_{0}$ ). We will prove that after any number of steps the covariance matrix sustains this structure. The discrete-time inverse Riccati equation for the propagation and update of the information matrix $\mathbf{P}^{-1}$ is:

$$
\begin{aligned}
\mathbf{P}_{k+1}^{-1} & =\left(\boldsymbol{\Phi}_{k} \mathbf{P}_{k} \boldsymbol{\Phi}^{T}{ }_{k}+\mathbf{G}_{k} \mathbf{Q G}_{k}^{T}\right)^{-1}+\mathbf{H}^{T} \mathbf{R}^{-1} \mathbf{H} \\
& =\left(\mathbf{P}_{k}+\mathbf{Q}\right)^{-1}+\mathbf{H}^{T} \mathbf{R}^{-1} \mathbf{H}
\end{aligned}
$$

where since the robots move independently it is $\boldsymbol{\Phi}_{k}=\mathbf{I}\left(I_{2 \times 2}\right)$ and $\mathbf{G}_{k}=\mathbf{I}\left(I_{2 \times 2}\right)$. Substituting $\mathbf{P}_{0}=\mathbf{I}\left(P_{0}\right)$ and $\mathbf{Q}=\mathbf{I}\left(\bar{Q}_{d}\right)$ we have: ${ }^{3}$

$$
\begin{aligned}
\mathbf{P}_{1}^{-1} & =\left(\mathbf{I}\left(P_{0}\right)+\mathbf{I}\left(\bar{Q}_{d}\right)\right)^{-1}+\mathbf{H}^{T} \mathbf{R}^{-1} \mathbf{H} \\
& =\mathbf{I}\left(\left(P_{0}+\bar{Q}_{d}\right)^{-1}+2 N^{o} \bar{R}^{-1}\right)+\mathbf{1}\left(-2^{o} \bar{R}^{-1}\right) \\
& =\mathbf{I}\left(A_{1}^{-1}\right)+\mathbf{1}\left(B_{1}^{\prime-1}\right)
\end{aligned}
$$

By employing the relations in Appendix A the covariance matrix can be computed as:

$$
\begin{aligned}
\mathbf{P}_{1} & =\mathbf{I}\left(A_{1}\right)+\mathbf{1}\left(-\left(A_{1}^{-1}+N B_{1}^{\prime-1}\right)^{-1} B_{1}^{\prime-1} A_{1}\right) \\
& =\mathbf{I}\left(A_{1}\right)+\mathbf{1}\left(B_{1}\right)
\end{aligned}
$$

Note again that both the diagonal and non-diagonal submatrix elements of this matrix are equal between them. Assume that after a certain number of propagation and update steps, at time step $k=m$, the covariance matrix has still equal diagonal and equal non-diagonal submatrix elements. That is

$$
\mathbf{P}_{m}=\mathbf{I}\left(A_{m}\right)+\mathbf{1}\left(B_{m}\right)
$$

We will prove that the covariance matrix $\mathbf{P}_{m+1}$ also has equal diagonal and non-diagonal submatrix elements. By substituting Eq. (18) in Eq. (17) we have:

$$
\begin{aligned}
\mathbf{P}_{m+1}^{-1}= & \left(\mathbf{I}\left(A_{m}\right)+\mathbf{1}\left(B_{m}\right)+\mathbf{I}\left(\bar{Q}_{d}\right)\right)^{-1}+\mathbf{H}^{T} \mathbf{R}^{-1} \mathbf{H} \\
= & \mathbf{I}\left(\left(A_{m}+\bar{Q}_{d}\right)^{-1}+2 N^{o} \bar{R}^{-1}\right) \\
& +\mathbf{1}\left(-\left(A_{m}+\bar{Q}_{d}+N B_{m}\right)^{-1} B_{m}\left(A_{m}+\bar{Q}_{d}\right)^{-1}-2{ }^{o} \bar{R}^{-1}\right) \\
= & \mathbf{I}\left(A_{m+1}^{-1}\right)+\mathbf{1}\left(B_{m+1}^{\prime-1}\right)
\end{aligned}
$$

${ }^{3} \bar{Q}_{d}$ is the discrete time equivalent of $\bar{Q}_{c}$, the continuous time covariance for the system propagation noise. 
By employing the relations in Appendix A once more, the covariance matrix can be computed as:

$$
\begin{aligned}
\mathbf{P}_{m+1} & =\mathbf{I}\left(A_{m+1}\right)+\mathbf{1}\left(-\left(A_{m+1}^{-1}+N B_{m+1}^{\prime-1}\right)^{-1} B_{m+1}^{\prime-1} A_{m+1}\right) \\
& =\mathbf{I}\left(A_{m+1}\right)+\mathbf{1}\left(B_{m+1}\right)
\end{aligned}
$$

We have proven the following:

LEMMA 3.1. The covariance matrix for a homogeneous ${ }^{4}$ group of robots, when they perform cooperative localization, is on the average a matrix with equal diagonal and equal non-diagonal submatrix terms.

A direct result of the previous lemma is the following:

COROLLARY 3.2. A homogeneous group of robots, when they perform cooperative localization, they experience the same level of positioning uncertainty and they share the same amount of information.

The amount of information shared by two robots is captured in the cross-correlation terms (non-diagonal submatrices) of the covariance matrix.

\section{Uncertainty Bounds for Cooperative Localization}

At this point we employ Lemma 3.1 to derive the main result of this paper, an analytical expression for the rate of increase in the localization uncertainty for a group of $N$ robots.

LEMMA 4.1. For a homogeneous group of $N$ robots, when they perform cooperative localization their covariance at steady state grows, on the average, linearly with time:

$$
\mathbf{P}_{i j}(t)=\left\{\begin{aligned}
\left(\frac{1}{N} q_{c} t+\frac{1}{N} p_{0}+\frac{N-1}{N} a_{c}\right) I_{2 \times 2}, i=j \\
\left(\frac{1}{N} q_{c} t+\frac{1}{N} p_{0}-\frac{1}{N} a_{c}\right) I_{2 \times 2}, i \neq j
\end{aligned}\right.
$$

with $i, j=1 \ldots N$ and

$$
a_{c}=\sqrt{\frac{q_{c}^{o} \bar{r}_{z}}{2 N}}
$$

\footnotetext{
${ }^{4}$ Homogeneous group of robots denotes a team comprised of robots with the same level of uncertainty for their proprioceptive and exteroceptive measurements.
} 
Proof In order to compute the time evolution of the covariance matrix for a group of $N$ robots, we employ the continuous time Riccati equation:

$$
\dot{\mathbf{P}}=\mathbf{F P}+\mathbf{P F} \mathbf{F}^{T}+\mathbf{G Q G}^{T}-\mathbf{P H}^{T} \mathbf{R}^{-1} \mathbf{H P}
$$

where $\mathbf{F}=\mathbf{I}\left(0_{2 \times 2}\right), \mathbf{G}=\mathbf{I}\left(I_{2 \times 2}\right), \mathbf{Q}=\mathbf{I}\left(\bar{Q}_{c}\right)$ and

$$
\begin{aligned}
\mathbf{H}^{T} \mathbf{R}^{-1} \mathbf{H} & =\mathbf{I}\left(2 N^{o} \bar{R}^{-1}\right)+\mathbf{1}\left(-2^{o} \bar{R}^{-1}\right), \\
\mathbf{P} & =\mathbf{I}(A)+\mathbf{1}(B)
\end{aligned}
$$

By substituting these terms in Eq. (21) it can be shown [28] that

$$
\dot{\mathbf{P}}=\mathbf{I}(\dot{A})+\mathbf{1}(\dot{B})=\mathbf{I}\left(\bar{Q}_{c}-2 N A^{o} \bar{R}^{-1} A\right)+\mathbf{1}\left(2 A^{o} \bar{R}^{-1} A\right)
$$

or

$$
\begin{aligned}
& \dot{A}(t)=\bar{Q}_{c}-2 N A(t){ }^{o} \bar{R}^{-1} A(t) \\
& \dot{B}(t)=2 A(t){ }^{o} \bar{R}^{-1} A(t)=\frac{1}{N}\left(\bar{Q}_{c}-\dot{A}(t)\right)
\end{aligned}
$$

with $A(0)=P(0)=p_{0} I_{2 \times 2}, B(0)=0_{2 \times 2}$.

If $A(t)$ was known, then matrix $B(t)$ can be determined from Eqs. (11) and (24):

$$
\begin{aligned}
B(t) & =\frac{1}{N}\left(\int_{0}^{t} \bar{Q}_{c} d \tau-\int_{0}^{t} \dot{A}(\tau) d \tau\right) \\
& =\frac{1}{N}\left(\left(q_{c} t+p_{0}\right) I_{2 \times 2}-A(t)\right)
\end{aligned}
$$

In Eq. (23), due to the structure of matrices $\bar{Q}_{c}$ (from Eq. (11)) and ${ }^{o} \bar{R}^{-1}$ (from Eq. (16)), $A(t)$ can be written as:

$$
A(t)=a(t) I_{2 \times 2}
$$

with $a(0)=p_{0}$. Substituting in Eq. (23), yields:

$$
\dot{a}(t)=q_{c}-2 N \frac{a^{2}(t)}{{ }^{o} \bar{r}_{z}}
$$

Solving this equation, at steady state, yields $\lim _{t \rightarrow \infty} a(t)=a_{c}$ with $a_{c}$ defined by Eq. (20). Using this result in Eqs. (26), (25), we have:

$$
A(t)=a_{c} I_{2 \times 2} \quad, \quad B(t)=\frac{1}{N}\left(q_{c} t+p_{0}-a_{c}\right) I_{2 \times 2}
$$

Finally, by substituting for $A(t)$ and $B(t)$ in Eq. (22), for the steady state value of $a(t)$ (Eq. (20)), the maximum expected covariance for a group of $N$ robots is computed by Eq. (19). 
COROLLARY 4.2. For a homogeneous group of $N$ robots performing cooperative localization, the maximum expected rate of positioning uncertainty increase at steady state is $\dot{P}_{i i}=\frac{1}{N} q_{c}$, i.e., it is inversely proportional to the number of robots $N$.

Proof Differentiation of Eq. (19) provides this result.

The following significant remarks are evident:

1. The rate of increase at steady state is inversely proportional to the number $N$ of robots and proportional to the odometric and orientation uncertainty of each robot (captured by $q_{c}$ ). The above result is also supported by the simulation study presented in [25] using a particle filter based estimator. Appropriate curve fitting calculated the rate of uncertainty increase proportional to $N^{-0.948}$.

2. The rate of uncertainty increase at steady state does not depend on the accuracy of the relative position measurements (captured by their covariance ${ }^{o} \bar{r}_{z}$ ).

3. The time for the cooperative localization system to reach steady state is determined by the time constant of the system related to the flow of information amongst the robots [28]:

$$
\tau=\frac{1}{2} \sqrt{\frac{{ }^{o} \bar{r}_{z}}{2 N q_{c}}}
$$

Inaccurate relative position measurements ${ }^{o} \bar{r}_{z}$ will delay the system reaching steady state. On the other hand large teams $N$ of robots with precise odometric and orientation information $q_{c}$ will quickly reach steady state.

Up to this point, we have assumed that all robots have the same odometric/orientation uncertainty $q_{c_{i}}=q_{c}$ and relative position measurement uncertainty ${ }^{\circ} \bar{r}_{z_{i}}={ }^{o} \bar{r}_{z}$. These assumptions were made in order to facilitate the previous derivations and thus gain insight into the structure of the cooperative localization problem. Nevertheless, Eq. (19) can be used as an inequality to determine the upper bound of the expected uncertainty growth with $q_{c}=\max \left(q_{c_{i}}\right),{ }^{o} \bar{r}_{z}=\max \left({ }^{o} \bar{r}_{z_{i}}\right)$. 


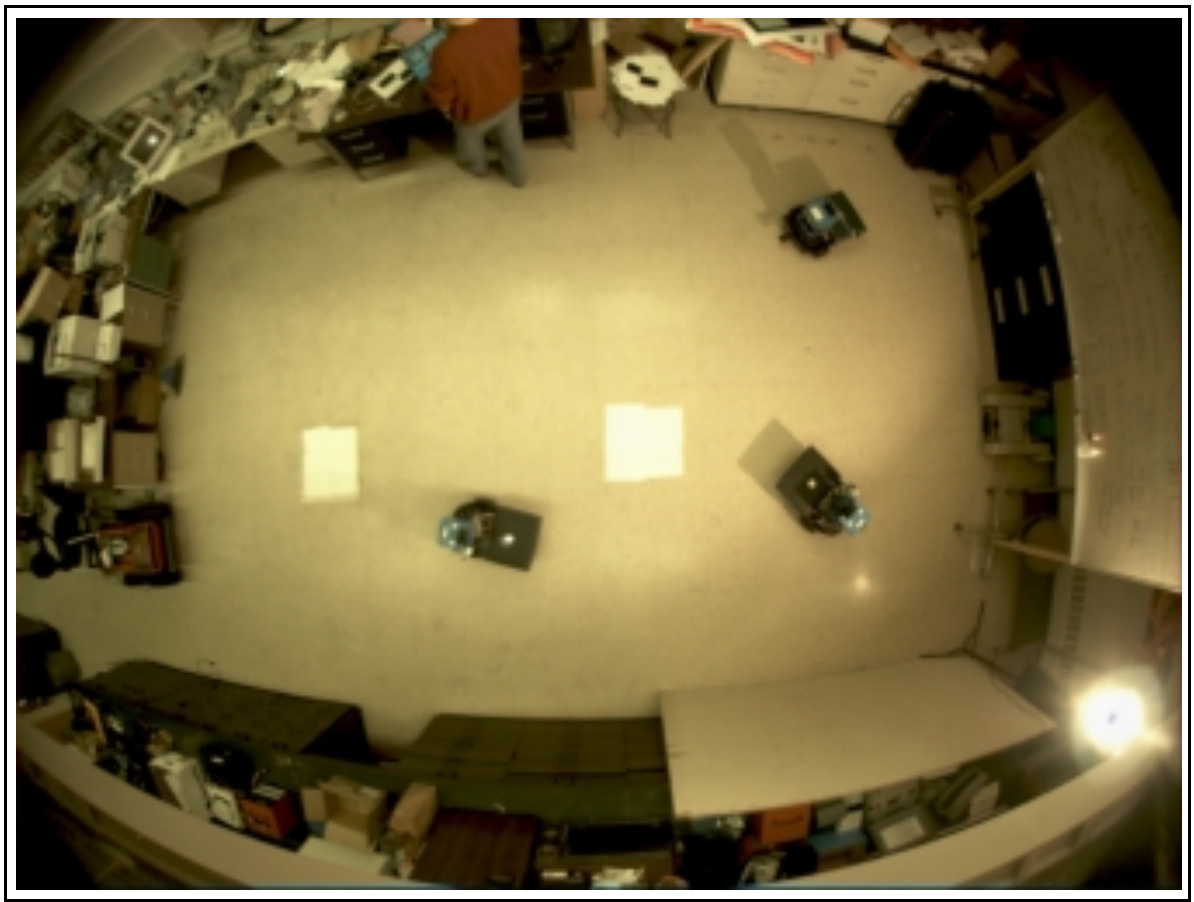

Figure 1. The Pioneer robots in the experimentation arena as viewed from the overhead camera.

\section{Experimental Results}

The cooperative localization algorithm was implemented and tested for the case of one (for comparing to dead reckoning), two, and three Pioneer mobile robots shown in Fig. 1. The experiments were conducted in a lab environment within an arena of dimensions $5.6 \mathrm{~m} \times$ $3.7 \mathrm{~m}$. The three robots started from different locations and were commanded to move randomly within this area, while avoiding obstacles, with constant linear velocity $V_{t}=0.1 \mathrm{~m} / \mathrm{sec}$. Every Pioneer robot is equipped with wheel-encoders on the two front wheels that measure the translational and rotational velocity of the vehicle. The standard deviation for the linear and rotational velocity measurements of the robots was experimentally determined to be $\sigma_{V}=0.005 \mathrm{~m} / \mathrm{sec}$ and $\sigma_{\omega}=0.0236 \mathrm{rad} / \mathrm{sec}$.

An overhead camera based tracking system was designed for recording the absolute pose (reference trajectory) of each of the three robots (Fig. 2). This ground truth was used for determining the errors $\varepsilon_{X}=$ $X_{\text {camera }}-\widehat{X}_{\mathrm{KF}}$ in the pose estimates $\widehat{X}_{K F}^{T}=[\hat{x} \hat{y} \hat{\phi}]$ of the Kalman Filter (KF). The accuracy of the tracking system is $2 \mathrm{~cm}$ for the position 


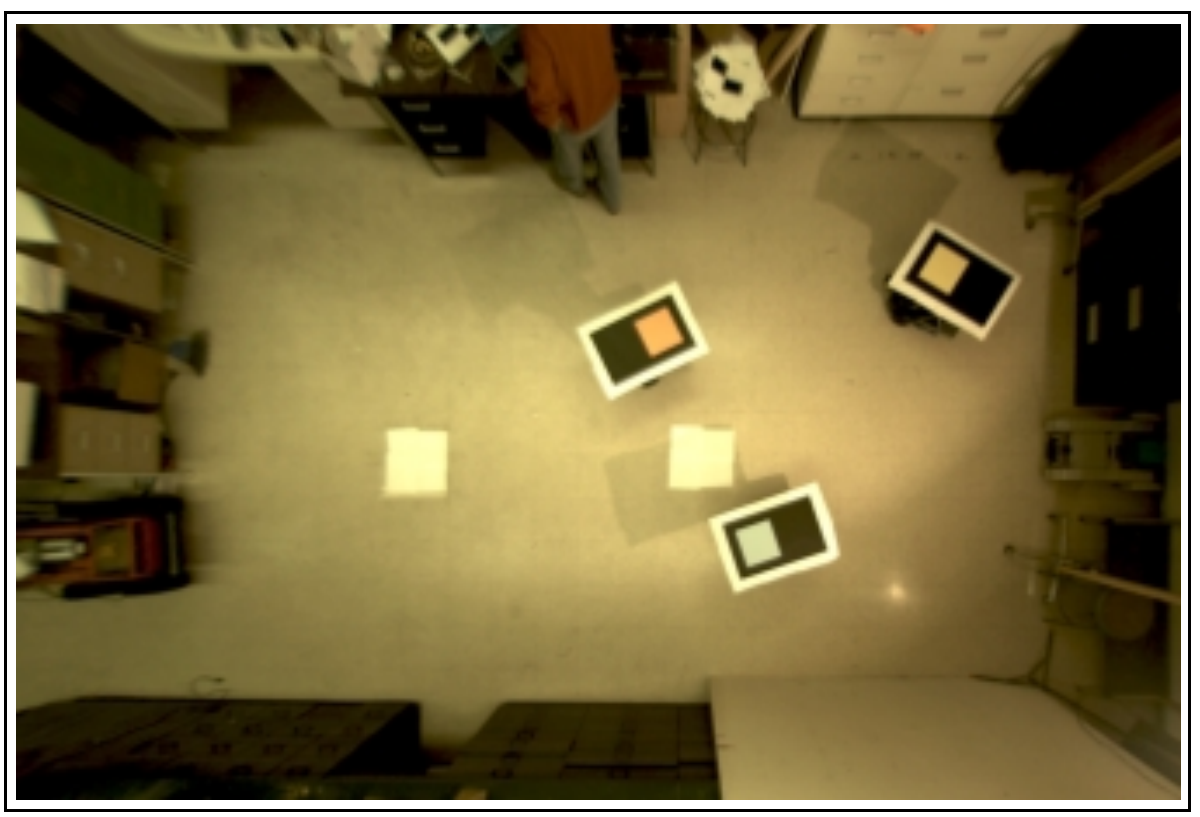

Figure 2. Rectified image of the Pioneer robots with color patches for tracking and identification as viewed from the overhead camera.

and $3^{\circ}$ for the orientation. In our experiments, instead of implementing a system that directly measures the relative position between two robots, such as the ones presented in [2,3], we use the information from the tracking system to infer these measurements. Each relative position measurement is the difference between the positions of two robots, expressed in local coordinates, with the addition of noise. This way we were able to investigate the validity of our theoretical analysis over a wide range of values for the accuracy level of the relative position sensor data and control the frequency of these measurements. For the experiments reported here the relative distance and bearing measurements had accuracy of $\sigma_{\rho}=0.1 \mathrm{~m}$ and $\sigma_{\theta}=0.2 \mathrm{rad}$ respectively. Finally, the absolute orientation measurements had accuracy $\sigma_{\phi}=0.0524 \mathrm{rad}$.

We have examined and present experimental results for three different scenarios (5 trials each): (i) Dead-reckoning (1 robot) - Fig. 3(a), (ii) Cooperative Localization (2 robots) - Figs. 3(c), (e), (iii) Cooperative Localization (3 robots) - Fig. 3(b), (d), (f). In each set of these figures, the following quantities are depicted:

- The position errors $\varepsilon_{x}$ in the estimates $\hat{x}_{\mathrm{KF}}$ along the $x$-axis for each robot and during sets of 5 experiments, as these were recorded by the overhead tracking camera (solid lines). 


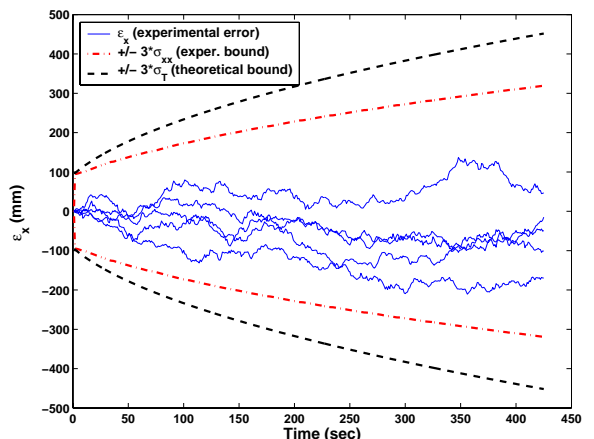

(a)

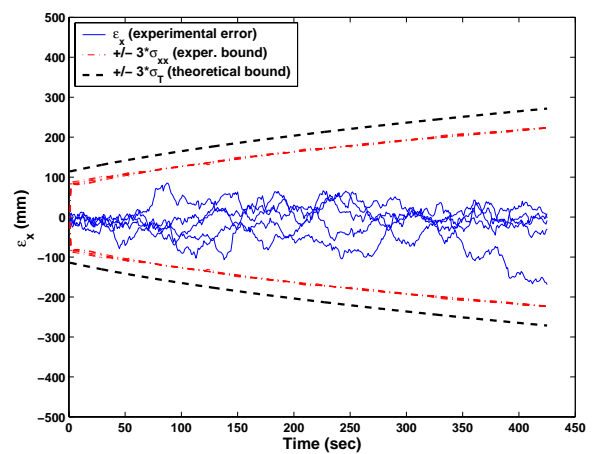

(c)

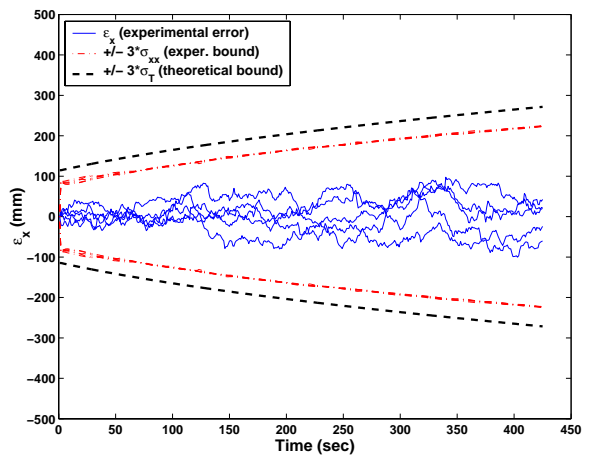

(e)

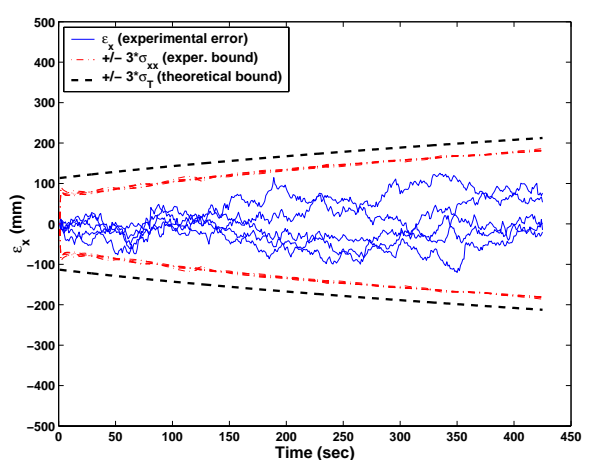

(b)

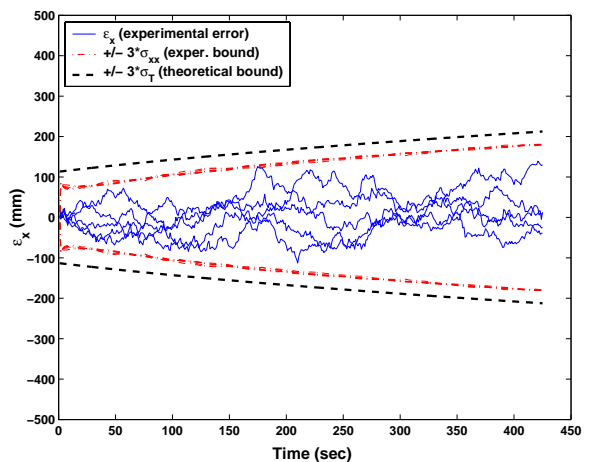

(d)

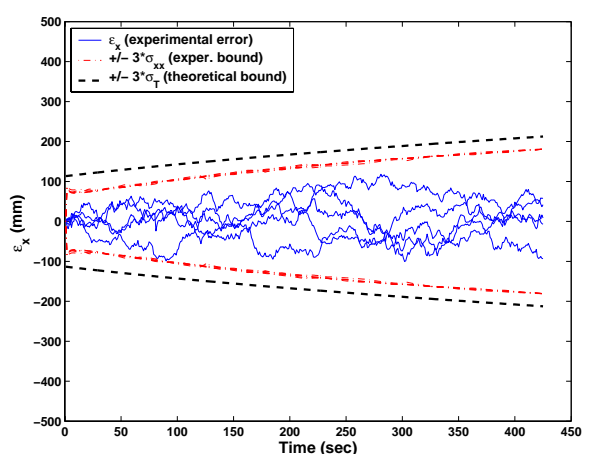

(f)

Figure 3. Position errors $\varepsilon_{x}$ (solid lines), experimentally calculated uncertainty bounds $\pm 3 \sigma_{x x}$ (dashed-dotted lines), and theoretically predicted maximum expected uncertainty bounds $\pm 3 \sigma_{T}$ as a function of time for the following cases (a) Robot \#1 from a 1-robot team (dead reckoning), (b) Robot \#1 from a 3-robot team. (c) Robot \#1 from a 2-robot team. (d) Robot \#2 from a 3-robot team. (e) Robot \#2 from a 2-robot team. (f) Robot \#3 from a 3-robot team. 
- The experimentally calculated, by the KF on each robot, $\pm 3 \sigma_{x x}=$ $\pm 3 \sqrt{P_{x x}}$ regions of confidence for each of these 5 trials (dasheddotted lines). These plots verify that in all cases examined, the KF estimator is consistent since the recorded errors $\varepsilon_{x}$ remain between the experimentally determined upper and lower bounds.

- The theoretically predicted $\pm 3 \sigma_{T}= \pm 3 \sqrt{P_{T}}$ regions of confidence for each of the 3 different scenarios examined (dashed lines). The values of $P_{T}$ are computed using the expression in Eq. (19). As it is evident these theoretically determined maximum expected values for the positioning uncertainty are in all cases enclosing those calculated by the KF. These bounds can be used a priori for determining bounds on the localization accuracy of teams of robots as a function of their size $N$ and the accuracy of their proprioceptive and exteroceptive measurements.

\section{Simulation Results}

In order to validate the results of the theoretical analysis for larger teams of robots $(N=2, \ldots, 5)$, we additionally performed a series of experiments in simulation. The setup used in these experiments is described hereafter. $N$ robots were placed inside a $40 \mathrm{~m} \times 40 \mathrm{~m}$ arena at random locations (see Fig. 4 for four robots). The robots were kept inside the designated area thus limiting the maximum inter-robot distance to the length of the diagonal. During the experiment the linear velocity of all robots was maintained constant $\left(V_{t}=0.25 \mathrm{~m} / \mathrm{sec}\right)$ while the rotational velocity was changed randomly as follows:

$$
\omega_{t}=\omega_{\max } \cdot n_{0,1}
$$

where $\omega_{\max }=0.2 \mathrm{rad} / \mathrm{sec}$ and $n_{0,1}$ is a random value drawn from the normal distribution with zero-mean and $\sigma=1$.

The simulated robot motion was recorded along with the dead reckoning (DR) and Kalman filter (KF) cooperative localization estimates. The velocity measurements were corrupted by additive zero-mean white Gaussian noise with noise parameters measured on a iRobot PackBot robot $\left(\sigma_{V}=0.01 \mathrm{~m} / \mathrm{sec}, \sigma_{\omega}=0.0384 \mathrm{rad} / \mathrm{sec}\right)$. The absolute orientation of each robot was measured by a simulated compass with $\sigma_{\phi}=0.0524 \mathrm{rad}$. The robot tracker sensor returned range and bearing measurements corrupted by zero-mean white Gaussian noise with $\sigma_{\rho}=0.01 \mathrm{~m}$ and $\sigma_{\theta}=0.0349 \mathrm{rad}$. The above values are compatible with noise parameters observed in laboratory experiments [26]. All measurements were available at $1 \mathrm{~Hz}$. The robots performed random walks for 10 


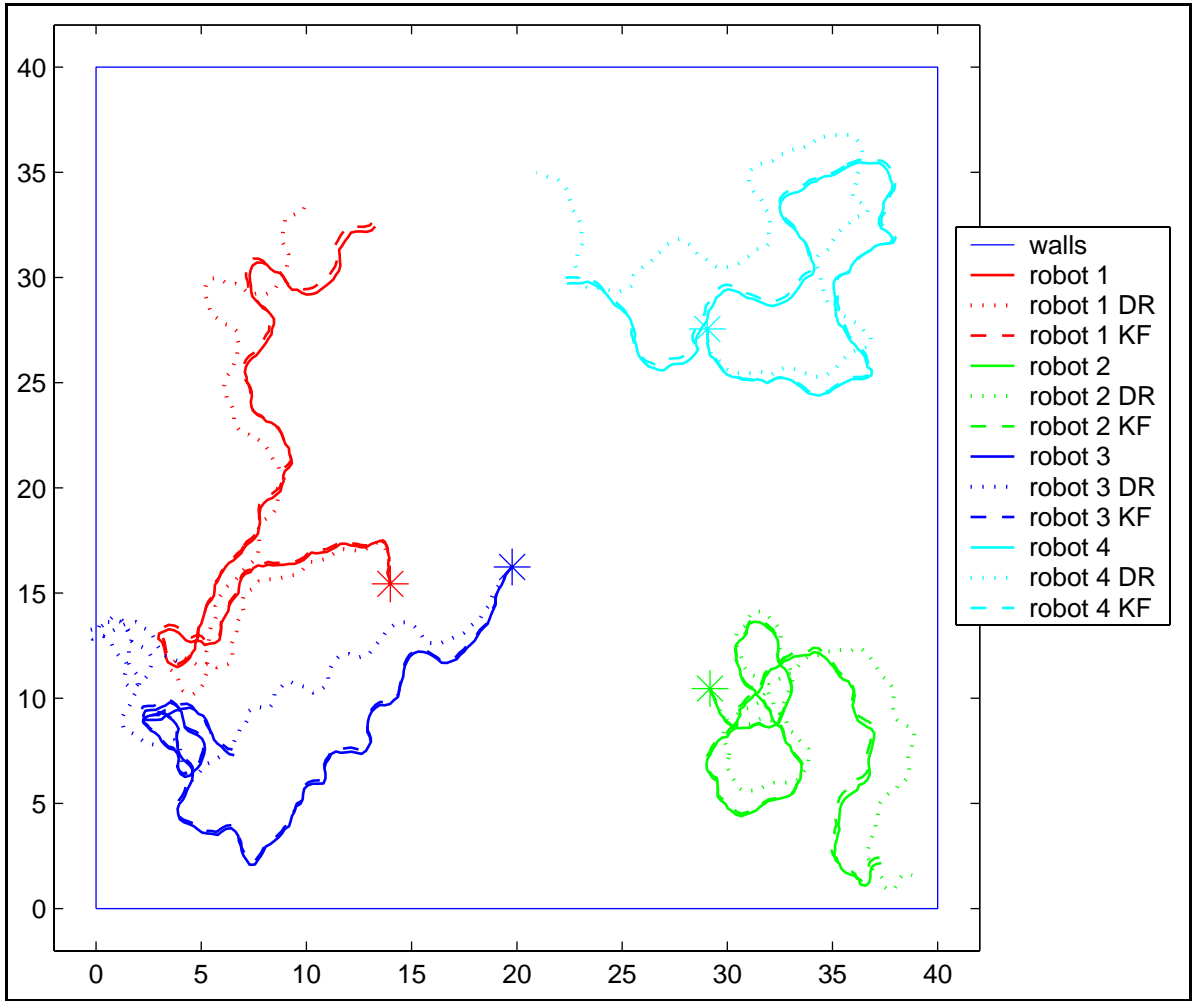

Figure 4. Four robots move randomly inside a $40 \mathrm{~m}$ by $40 \mathrm{~m}$ arena. Starting positions are marked by "*”.

min at each trial while continuously measuring their relative positions. Fig. 4 presents the ground truth (solid lines), the dead reckoning based trajectories (dotted lines) and the KF (dashed lines) for four robots from one trial.

Fig. 5 depicts the comparison between the theoretically derived upper bound from the analytical expression in Eq. (19) (dashed lines) and the average, across $N$ robots, covariance along the $x$ and $y$ axes for ten different trials (solid lines). Each figure (5(a)-5(d)) presents the results for an increasing number of robots $(N=2, \ldots, 5)$ and consists of two sub-plots: the top one for the $x$-axis and the bottom one for the $y$-axis. As evident the average covariance values $\left(P_{x x}, P_{y y}\right)$ are consistently lower compared to the theoretically derived upper bound, and follow (on average) the same rate of increase.

It is worth noting that as the number $N$ of robots increases, the constant offset between the theoretically derived covariance upper bound and the recorded average covariance grows larger. This is due to the fact 

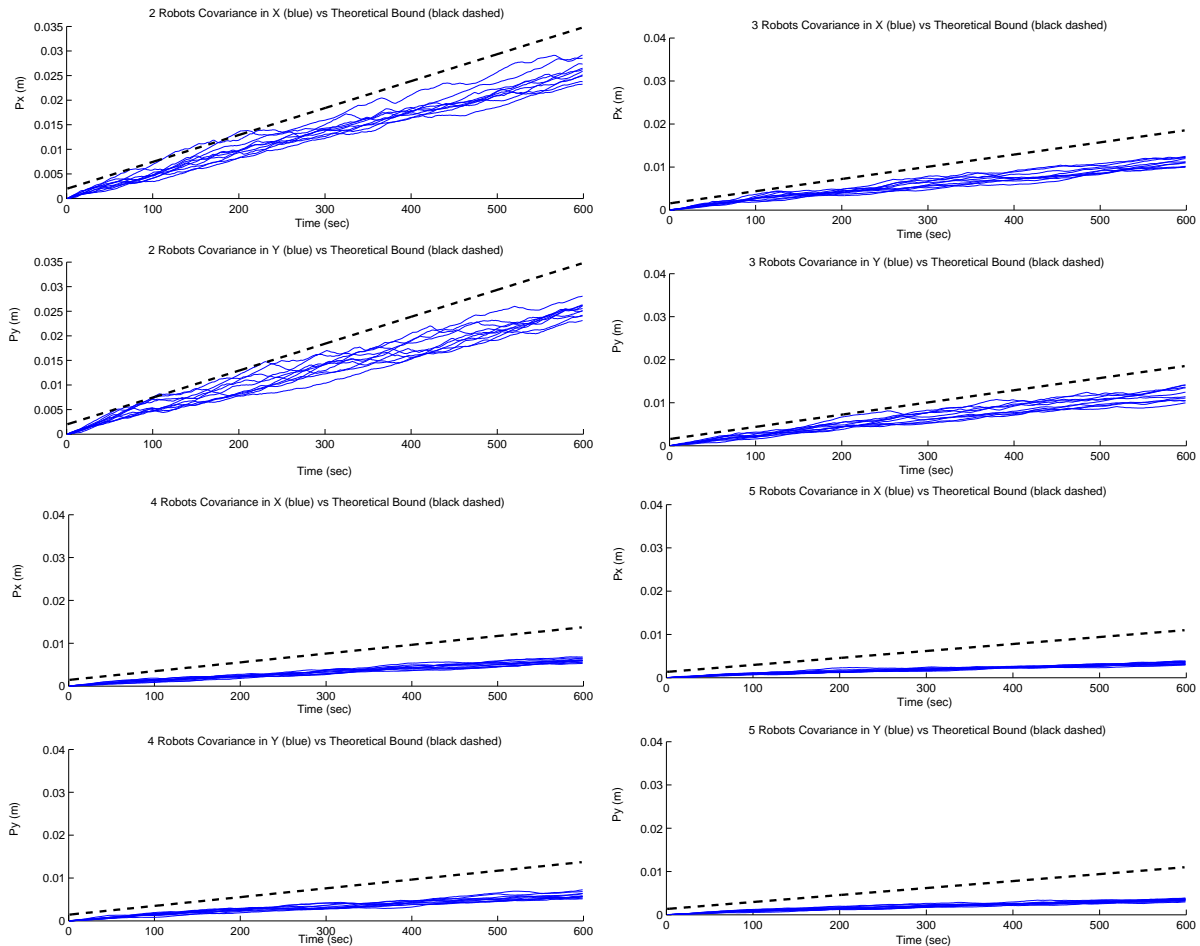

Figure 5. The covariance along $x$-axis and $y$-axis for ten experiments (solid thin lines) and the theoretical upper bound of uncertainty (dashed lines) for different number of robots $(N=2, \ldots, 5)$.

that the parameter $\rho_{o}$ (maximum average distance between the robots) decreases significantly as the number of robots populating the same area increases. This in effect reduces the constant term in the linear, with respect to time, expression for the maximum expected covariance in Eq. (19).

\section{Conclusions}

This paper presented a theoretical analysis for the propagation of position uncertainty for a team of mobile robots. One of the most challenging cases of localization was considered where inter-robot observations (cooperative localization), dead reckoning estimates, and absolute orientation measurements were available. Furthermore, all robots moved simultaneously, in contrast to previous work where often the robots take turns to move and to act as landmarks. An analytical formula was derived that expresses the upper bound of the uncertainty accumulation as a function of time and the noise characteristics of the robot sensors. 
The provable upper bound on the rate of position uncertainty increase in Eq. (19) allows to draw the following important conclusions. First, the uncertainty growth is inversely proportional to the number of robots thus the contribution of each additional robot follows a law of diminishing return. Second, the rate of uncertainty growth depends only on the number of robots and the odometric and orientation uncertainty and not on the accuracy of the relative position measurements. These results were verified experimentally and in simulation for robot groups of increasing size. The estimated by the Kalman filter uncertainty, consistently remained below the theoretical upper bound.

From the form of the derivations we are confident that these results can be extended to the case of motion in $3 \mathrm{D}$, thus providing upper bounds for the position uncertainty of outdoor ground robots or autonomous aerial vehicles. Finally, we intend to study the effect on cooperative localization of different sensing modalities, motion strategies and robot formations.

\section{Acknowledgements}

This work was supported by the University of Minnesota (GiA Award, DTC), the Jet Propulsion Laboratory (Grant No. 1248696, 1251073), and the National Science Foundation (ITR, Grant No. EIA-0324864).

\section{References}

1. Y. Arai, T. Fujii, H. Asama, H. Kaetsu, and I. Endo. Realization of autonomous navigation in multirobot environment. In Proceedings of the 1998 IEEE/RSJ International Conference on Intelligent Robots and Systems, pages 1999-2004, Victoria, B.C., Canada, Oct. 13-17 1998.

2. W. Burgard, D. Fox, M. Moors, R. Simmons, and S. Thrun. Collaborative multi-robot exploration. In Proceedings of the IEEE International Conference on Robotics and Automation, pages 476-481, San Francisco, CA, Apr. 24-28 2000.

3. A.J. Davison and N. Kita. Active visual localisation for cooperating inspection robots. In Proceedings of the IEEE/RSJ International Conference on Intelligent Robots and Systems, pages 1709-15, Takamatsu, Japan, Oct. 31-Nov. 5 2000.

4. G. Dudek, M. Jenkin, E. Milios, and D. Wilkes. A taxonomy for multi-agent robotics. Autonomous Robots, 3:375-397, 1996.

5. C. Ferrari, E. Pagello, J. Ota, and T. Arai. Multirobot motion coordination in space and time. Robotic Autonomous Systems, 25(3-4):219-229, 1998.

6. M.S. Fontan and M.J. Mataric. Territorial multirobot task division. IEEE Transactions of Robotics and Automation, 14:815-822, 1998. 
7. D. Fox, W. Burgard, H. Kruppa, and S. Thrun. Collaborative multi-robot localization. In In Proceedings of the 23rd Annual German Conference on Artificial Intelligence (KI), pages 255-266, Bonn, Germany, Sep. 13-15 1999.

8. D. Fox, W. Burgard, H. Kruppa, and S. Thrun. A probabilistic approach to collaborative multi-robot localization. Autonomous Robots, 8(3):325-344, Jun. 2000. Special Issue on Heterogeneous Multirobot Systems.

9. D. W. Gage. Minimum resource distributed navigation and mapping. In Proceedings of SPIE Mobile Robots XV, volume 4195, 2000.

10. R. Grabowski and P. Khosla. Localization techniques for a team of small robots. In Proceedings of the IEEE/RSJ International Conference on Intelligent Robots and Systems, pages 1067-1072, Maui, HI, Oct.29-Nov.3 2001.

11. R. Grabowski, L. E. Navarro-Serment, C. J. J. Paredis, and P.K. Khosla. Heterogenouse teams of modular robots for mapping and exploration. Autonomous Robots, 8(3):293-308, 2000.

12. A. Howard, M.J. Mataric, and G.S. Sukhatme. Localization for mobile robot teams using maximum likelihood estimation. In Proceedings of the IEEE/RSJ International Conference on Intelligent Robots and Systems, pages 434-59, Lauzanne, Switzerland, Sep.30-Oct.4 2002.

13. K. Kato, H. Ishiguro, and M. Barth. Identifying and localizing robots in a multirobot system environment. In Proceedings of the IEEE/RSJ International Conference on Intelligent Robots and Systems, pages 966 - 971, Kyongju, South Korea, Oct. 17-21 1999.

14. A. Kelly. General solution for linearized systematic error propagation in vehicle odometry. In Proceedings of the IEEE/RSJ International Conference on Robots and Systems (IROS), pages 1938-45, Maui, HI, Oct.29-Nov.3 2001.

15. R. Kurazume and S. Hirose. Study on cooperative positioning system: optimum moving strategies for CPS-III. In Proceedings of the IEEE International Conference in Robotics and Automation, pages 2896-2903, Leuven, Belgium, May 16-20 1998.

16. R. Kurazume and S. Hirose. An experimental study of a cooperative positioning system. Autonomous Robots, 8(1):43-52, Jan. 2000.

17. R. Kurazume, S. Hirose, S. Nagata, and N. Sashida. Study on cooperative positioning system (basic principle and measurement experiment). In Proceedings of the IEEE International Conference in Robotics and Automation, pages 1421-1426, Minneapolis, MN, Apr. 22-28 1996.

18. R. Kurazume, S. Nagata, and S. Hirose. Cooperative positioning with multiple robots. In Proceedings of the IEEE International Conference in Robotics and Automation, pages 1250-1257, Los Alamitos, CA, May 8-13 1994.

19. F. Lu and E. Milios. Robot pose estimation in unknown environments by matching $2 \mathrm{~d}$ range scans. Journal of Intelligent and Robotic Systems: Theory and Applications, 18(3):249-275, Mar. 1997.

20. L. E. Parker. Current state of the art in distributed autonomous mobile robotics. In Proceedings of the 5th International Symposium on Distributed Autonomous Robotic Systems (DARS 2000), pages 3-12, Knoxville, TN, Oct. 4-6 2000.

21. L.E. Parker. Alliance: An architecture for fault tolerant multirobot cooperation. IEEE Transactions on Robotics and Automation, 220-240, 1998.

22. S.T. Pfister, K.L. Kriechbaum, S.I. Roumeliotis, and J.W. Burdick. Weighted range matching algorithms for mobile robot displacement estimation. IEEE Transactions on Robotics and Automation, 2003. (submitted). 
23. I.M. Rekleitis, G. Dudek, and E. Milios. Multi-robot exploration of an unknown environment, efficiently reducing the odometry error. In Proceedings of the Fifteenth International Joint Conference on Artificial Intelligence (IJCAI'97), pages 1340-1345, Nagoya, Japan, Aug. 23-29 1997.

24. I.M. Rekleitis, G. Dudek, and E. Milios. Multi-robot collaboration for robust exploration. Annals of Mathematics and Artificial Intelligence, 31(1-4):7-40, 2001.

25. I.M. Rekleitis, G. Dudek, and E. Milios. Multi-robot cooperative localization: A study of trade-offs between efficiency and accuracy. In Proceedings of the IEEE/RSJ International Conference on Intelligent Robots and Systems, Lausanne, Switzerland, Sep.30-Oct.4 2002.

26. I.M. Rekleitis, G. Dudek, and E. Milios. Probabilistic cooperative localization in practice. In Proceedings of the IEEE International Conference on Robotics and Automation, pages 1907-1912, Taipei, Taiwan, Sep. 14-19 2003.

27. S.I. Roumeliotis. Distributed Multi-Robot Localization. Technical report, California Institute of Technology, 2002. http://robotics.caltech.edu/ ${ }^{\sim}$ stergios/tech_reports/tr_collective.pdf.

28. S.I. Roumeliotis. Analytical expressions for multi-robot localization. Technical report, Computer Science and Engineering Department, University of Minnesota, Feb. 2003. http://www.cs.umn.edu/〜stergios/tech_reports/multi_analysis.pdf.

29. S.I. Roumeliotis and G.A. Bekey. Collective localization: A distributed kalman filter approach to localization of groups of mobile robots. In Proceeding of the IEEE International Conference on Robotics and Automation, pages 2958-2965, San Francisco, CA, Apr. 24-28 2000.

30. S.I. Roumeliotis and G.A. Bekey. Distributed multirobot localization. IEEE Transactions on Robotics and Automation, 18(5), Oct. 2002.

31. A.C. Sanderson. A distributed algorithm for cooperative navigation among multiple mobile robots. Advanced Robotics, 12(4):335-349, 1998.

32. S. Thrun, D. Fox, and W. Burgard. Monte carlo localization with mixture proposal distribution. In Proceedings of the AAAI National Conference on Artificial Intelligence, pages 859-865, Austin, TX, 2000.

33. W. Williamson, J. Min, J. L. Speyer, and J. Farrell. A comparison of state space, range space, and carrier phase differential GPS/INS relative navigation. In Proceedings of the American Control Conference, pages 2932-2938, Chicago, IL, June 2000.

\section{Appendix}

\section{A. Special Form of Matrix Inverse}

LEMMA A.1. The inverse of a matrix $\mathbf{C}=\mathbf{I}(A)+\mathbf{1}(B)$ that is comprised of $N$ equal invertible diagonal submatrices $\left(\exists B^{-1}\right)$ and $N(N-1)$ equal invertible non-diagonal submatrices $\left(\exists(A+N B)^{-1}\right)$, with $N \geq 2$ is $\mathbf{C}^{-1}=\mathbf{I}\left(A^{-1}\right)-\mathbf{1}\left((A+N B)^{-1} B A^{-1}\right)$.

Proof Multiplying the previous two matrices and employing the relation $\mathbf{1}(X) \mathbf{1}(Y)=\mathbf{1}(N X Y)$ yields $\mathbf{C C}^{-1}=\mathbf{I}(I)$ 